\title{
Influence of Regional Cerebral Blood Flow on Event-related Potential (P300)
}

\author{
Toshikazu Kuwata, Kazuyoshi FUnAHASHI, Shinichiro MAESHIMA, \\ Mitsuhiro OGURA, Genhachi HYOTANI, Tomoaki TERADA, \\ Toru ITAKURA, Seiji HAYASHI and Norihiko KOMAI
}

Department of Neurological Surgery, Wakayama Medical College, Wakayama

\begin{abstract}
The relationships between event-related potential (P300), higher brain functions, and regional cerebral blood flow (rCBF) were examined in 31 neurosurgical patients. The P300 latency, evaluated by an acoustic "odd-ball" paradigm, was normal in 14 and prolonged in 17 patients. Fourteen of 17 prolonged P300 patients had reduced rCBF in the right cerebral hemisphere, especially the frontal lobe and/ or thalamus. There was a significant inverse relationship between P300 latency and laterality index (rt $\mathrm{rCBF} / \mathrm{lt} \mathrm{rCBF}$ ) in the frontal lobe. Prolonged P300 patients revealed significant abnormalities in psychological tests compared to normal $\mathrm{P} 300$ patients. There were significant inverse relationships between the P300 latency and "orientation" and "memory" test scores. Decreased rCBF in the right cerebral hemisphere, especially the frontal lobe and/or thalamus, is associated with prolonged P300 latency, suggesting that the right cerebral hemisphere is important in human cognitive processes.
\end{abstract}

Key words: P300, cerebral blood flow, brain function

\section{Introduction}

Cognitive or emotional impairments may occur in patients disturbing their capacity for work and social reintegration. The $\mathbf{P} 300$ is an event-related potential that reflects human cognitive processes and has been used to examine higher brain functions in neurological ${ }^{1,7)}$ and neuropsychological disorders. ${ }^{6,12)}$

The origin of the $\mathbf{P} 300$ potential is controversial, but may be various neural structures including the parietal ${ }^{14)}$ or frontal association cortex, ${ }^{10)}$ hippocampus, ${ }^{11)}$ or thalamus. ${ }^{17)}$ Little is known about the influence of cerebral blood flow on the P300 in humans. ${ }^{16)}$

In this study, we examined the $\mathrm{P} 300$ potential and higher brain functions in neurosurgical patients and investigated the relationship between the regional cerebral blood flow (rCBF) and the human P300 potential.

Received April 10, 1992; 1992

\section{Materials and Methods}

Thirty-one right-handed patients (15 females and 16 males) underwent pre- or postoperative analysis of P300 potential and rCBF. Twenty-four patients had cerebrovascular diseases (13 hypertensive intracerebral hemorrhage, 6 arteriovenous malformation, 4 cerebral infarction, and 1 cerebral aneurysm), six had brain tumors ( 3 glioma and 3 meningioma), and one had hydrocephalus. Ages ranged from 25 to 81 years (mean, 55.1 yrs). Patients with impaired hearing or communication due to aphasia or severe dementia were excluded. All patients had unilateral lesions (17 right and 14 left hemispheres) on computed tomography (CT) (Fig. 1). The P300 potential and $\mathrm{rCBF}$ were also measured in 27 and eight normal subjects, respectively, to determine the normal range (mean $\pm 2 \mathrm{SD}$ ).

P300 potentials were recorded from the central $(\mathrm{Cz})$ and parietal $(\mathrm{Pz})$ locations (International 10-20 System), referenced to linked-earlobes using an "odd-ball" paradigm. The subjects were asked to count silently the rare tones $(2000 \mathrm{~Hz}$; probability $=$ 0.2 ) occurring at random among non-target events 

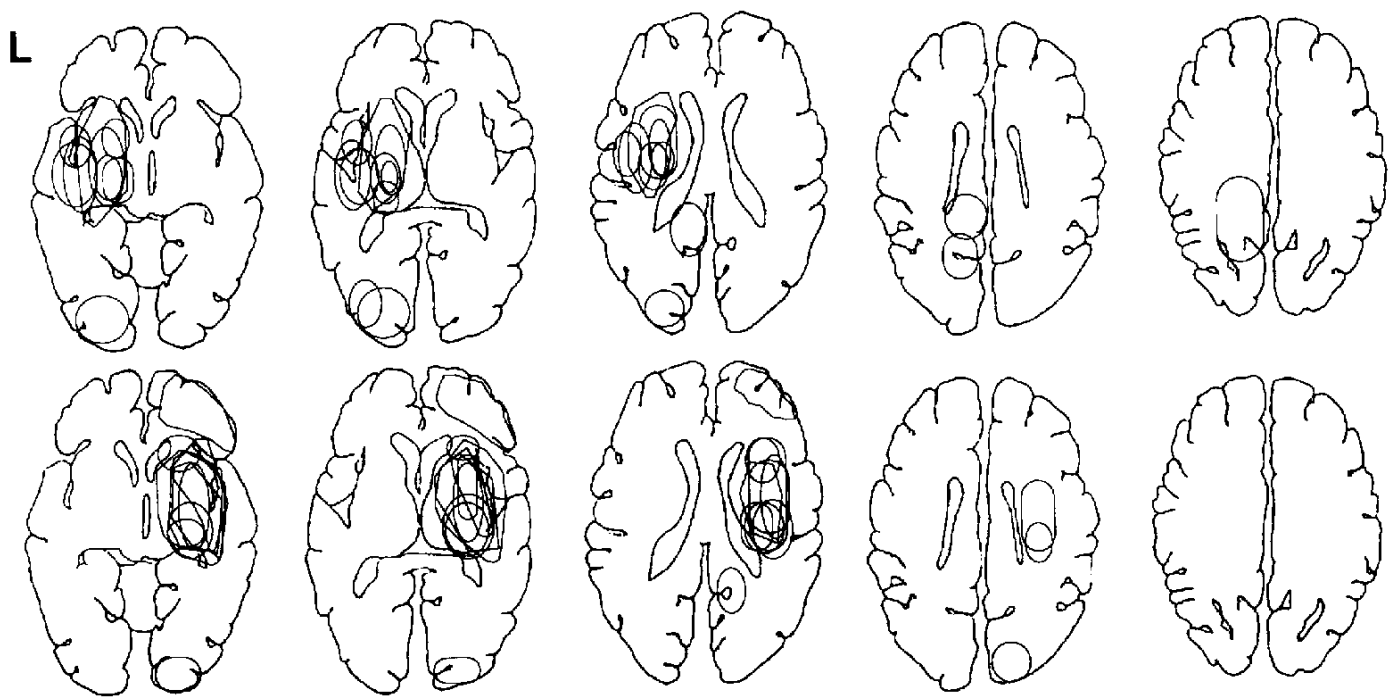

Fig. 1 Size and location of the lesions defined by CT at various levels. upper: left hemispheric lesions, lower: right hemispheric lesions.

$(1000 \mathrm{~Hz}$; probability $=0.8)$. All subjects could hear the tones clearly and distinguish the two pitches. Counting errors never exceeded two for normal subjects and five for patients. Thirty potentials were averaged separately following target and non-target stimuli (bandpass, $0.1-1000 \mathrm{~Hz}$; analysis time, $-100-900 \mathrm{msec}$ ). The response to rare tones was defined as a large positive peak with a peak latency of $300 \mathrm{msec}$ or more.

rCBF was measured by the cold xenon CT method. The mean interval between the P300 and rCBF measurements was 5 days. Following one control scan, five serial scans were made after inhalation of $35 \%$ cold xenon gas. Seven more scans were made after stopping xenon inhalation. Regions of interest (ROIs) were selected symmetrically in the frontal, temporal, and occipital lobes, thalamus, and basal ganglia. rCBF was evaluated with a laterality index (LI), defined as:

\section{$\mathrm{LI}=$ right $\mathrm{rCBF} /$ left $\mathrm{rCBF}$}

Reduction in rCBF was defined as deviation from the mean $\mathrm{LI} \pm 2 \mathrm{SD}$ in the eight normal subjects.

Four psychological tests were performed on the same day as the P300 measurement, including the Rating Scale for Psychological Function by Sano et al., ${ }^{13)}$ Mini-mental State, ${ }^{2}$ Hasegawa's Dementia Scale, ${ }^{8}$ and the Kanahiroi test. These tests all assess a specific aspect of psychological function. For example, the Rating Scale for Psychological Function mainly evaluates the spontaneity and activity of the patients, whereas the Mini-mental State emphasizes verbal function. The Kanahiroi test is designed for Japanese patients, who have to locate five vowels within 2 minutes in a story written in Japanese characters.

\section{Results}

There was a significant relationship between the P300 latency and age in 27 normal subjects ( $p<$ 0.005 ) (Fig. 2). The P300 latencies of the patients were divided into two groups: normal (14 cases with a mean latency of $353.1 \pm 22.2 \mathrm{msec}$ ) and prolong-

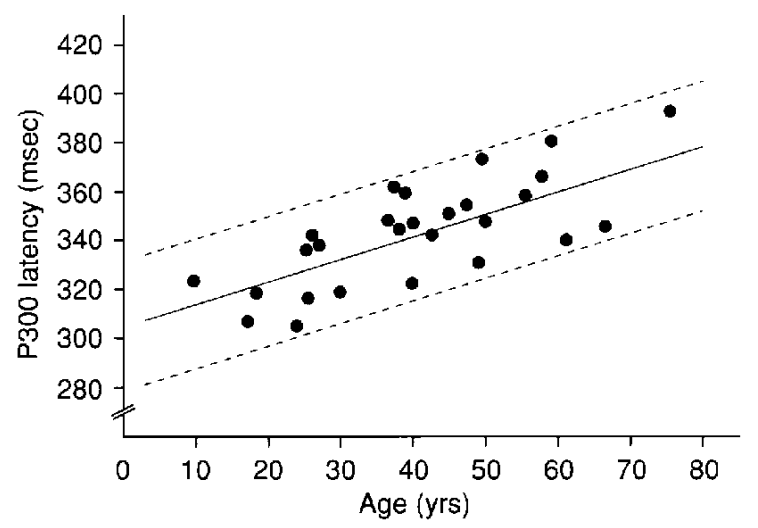

Fig. 2 P300 latency and age in 27 normal subjects. There was a significant relationship $(\mathrm{Y}=$ $0.972 X+303.9, \quad r=0.7501, \quad p<0.005)$. Solid and interrupted lines indicate mean and $\pm 2 \mathrm{SD}$, respectively ( $\mathrm{SD}=14.4 \mathrm{msec}$ ). 


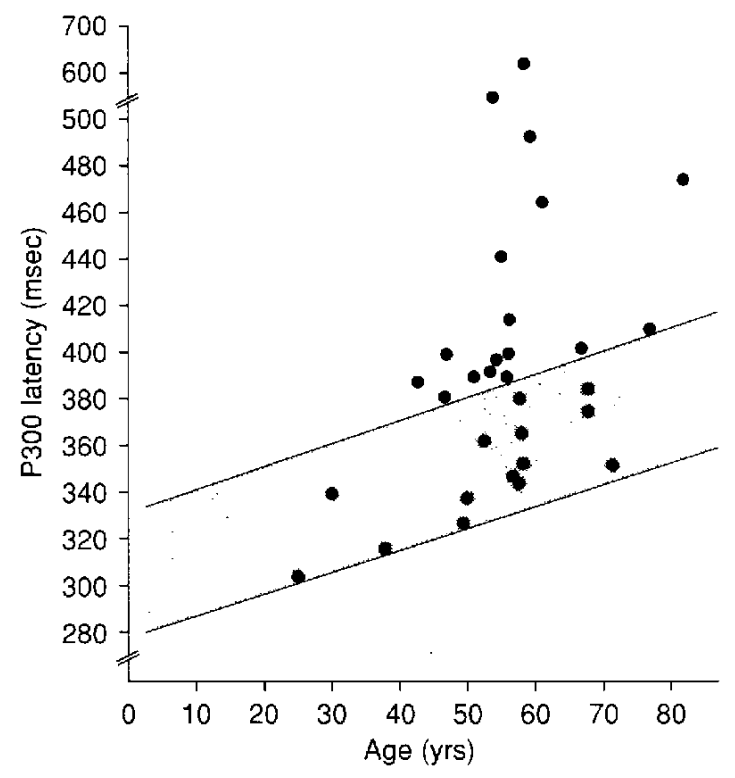

Fig. 3 P300 latency and age in 31 patients. The P300 latencies were normal in 14 cases and prolonged in 17. Shaded area represents the normal range (mean $\pm 2 \mathrm{SD}$ in normal subjects).

ed (17 cases with a mean latency of $444.1 \pm 72.5$ msec) (Fig. 3).

Eight $(57.1 \%)$ of the 14 patients with a normal P300 latency and $16(94.1 \%)$ of the 17 patients with a prolonged $\mathrm{P} 300$ latency had reduced $\mathrm{CCBF}$ in at least one or more ROIs (Table 1). All except one of the eight normal P300 patients demonstrated reduced rCBF in the left cerebral hemisphere. In contrast, 14 of the 16 prolonged $\mathrm{P} 300$ patients had reduced rCBF in the right cerebral hemisphere, mostly localized in the frontal lobe $(53.8 \%)$ and/or thalamus $(76.5 \%)$ (Fig. 4).

The LIs of the prolonged P300 patients were
Table 1 Results of rCBF and P300 studies in 31 patients

\begin{tabular}{lcc} 
& \multicolumn{2}{c}{ P300 latency } \\
\cline { 3 - 3 } rCBF & Normal & Prolonged \\
\hline Normal & 6 & 1 \\
Reduced & 8 & 16 \\
right side & 1 & 14 \\
left side & 7 & 2 \\
\hline
\end{tabular}

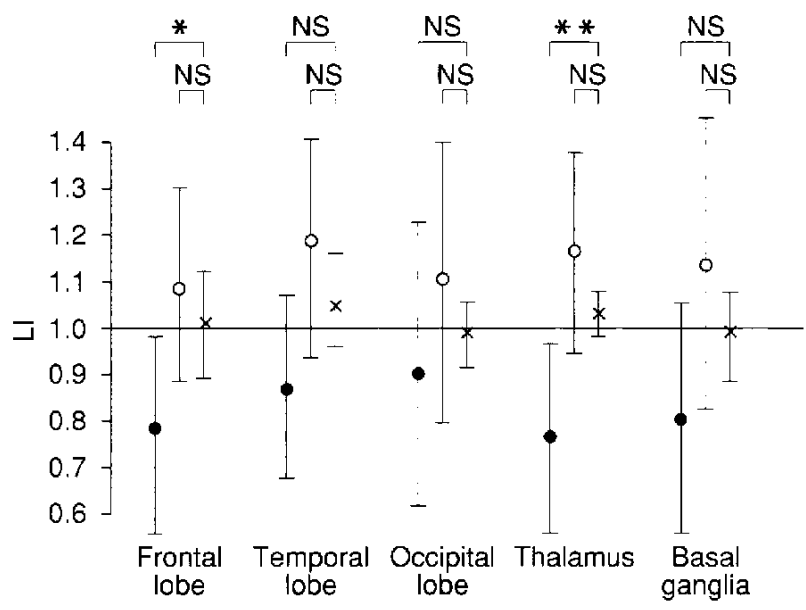

Fig. 5 LI in normal $(O)$ and prolonged P300 patients $(\bullet)$, and in normal subjects $(\times)$. In the frontal lobe and thalamus, the LIs of the prolonged P300 patients were significantly lower than those of normal subjects $\left({ }^{*} \mathrm{p}<0.05,{ }^{* *} \mathrm{p}<\right.$ $0.01)$. NS: not significant.

significantly lower in the frontal lobe $(0.79 \pm 0.21$; $\mathrm{p}<0.05)$ and thalamus $(0.76 \pm 0.22 ; \mathrm{p}<0.01)$ compared to the normal subjects (frontal lobe,

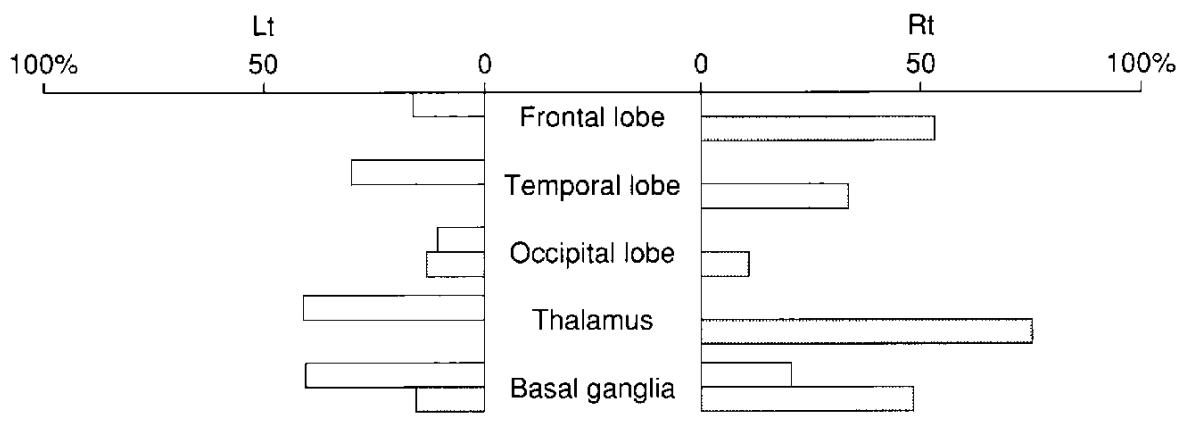

Fig. 4 Side and location of reduced rCBF in normal (open column) and prolonged P300 patients (shaded column). Most prolonged $\mathrm{P} 300$ patients had reduced rCBF in their right cerebral hemisphere, particularly the frontal lobe and/or thalamus. 


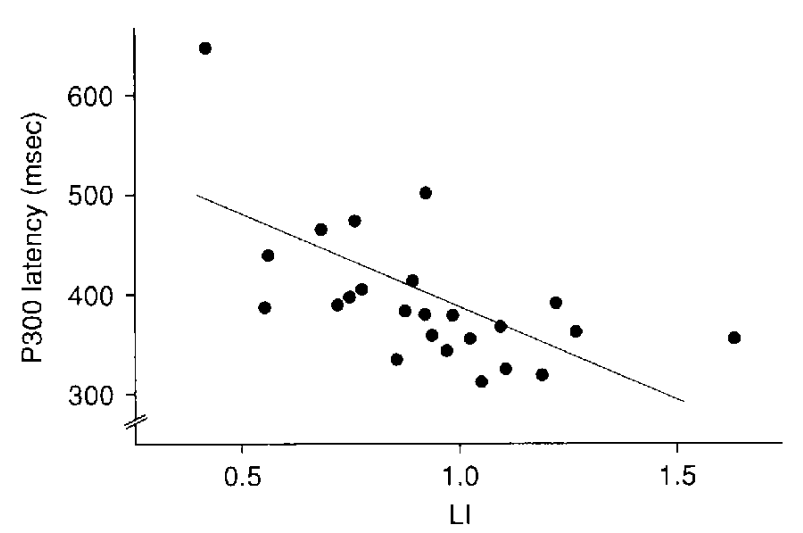

Fig. 6 P300 latency and the LI for the frontal lobe of the prolonged $\mathbf{P} 300$ patients. There was a significant inverse relationship $(\mathrm{Y}=574.74-$ 183.71X, $\mathrm{r}=-0.6030, \mathrm{p}<0.01)$.

$1.01 \pm 0.13 ;$ thalamus, $1.03 \pm 0.06$ ) (Fig. 5) Moreover, there was a significant inverse relationship between the P300 latency and the LI in the frontal lobe $(p<0.01)$ (Fig. 6).

The prolonged $\mathrm{P} 300$ patients revealed significant abnormalities on the Mini-mental State and Hasegawa's Dementia Scale when compared to normal P300 patients (Fig. 7). In subtests of cognitive performance, the prolonged $\mathrm{P} 300$ patients had significantly lower scores in "orientation" and "memory" (Fig. 8). There were significant correlations between P300 latency and patient scores in these two subtests (Fig. 9).

\section{Discussion}

The human P300 is a positive event-related potential with a latency of $300 \mathrm{msec}$ or greater, typically elicited by rare target stimuli in a detection task. Since the P300 potential appears to be related to human cognitive processes, it has been used to evaluate the higher brain functions of neurological or neuropsychological patients. Ebner et al. ${ }^{1)}$ reported that $\mathbf{P} 300$ abnormalities in patients with focal brain lesions correlated significantly with impairments detected by some psychological tests. Goodin et al. ${ }^{5}$ reported that $\mathrm{P} 300$ latency correlates with the Mini-mental State score in patients with dementia. Similarly, our study showed that patients with prolonged P300 latency caused by focal brain lesions have significant abnormalities detected by the Mini-mental State and Hasegawa's Dementia Scale tests. We also found that a prolonged P300 latency correlates significantly with disturbances in orientation and memory.

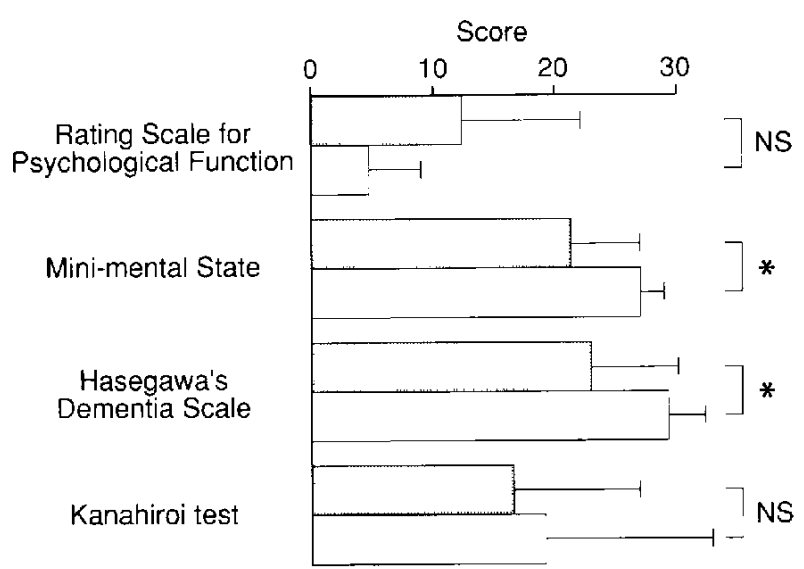

Fig. 7 Scores in cognitive performance tests for normal (open column) and prolonged P300 patients (shaded column). The prolonged P300 patients had significant abnormalities detected by Mini-mental State and Hasegawa's Dementia Scale tests compared to normal P300 patients $\left({ }^{*} p<0.05\right)$. A higher score indicates greater abnormality in the Rating Scale for Psychological Function.

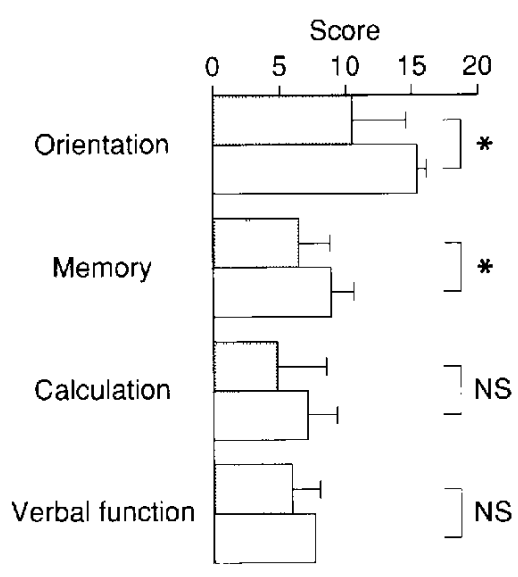

Fig. 8 Scores in cognitive subtests for normal (open column) and prolonged $\mathrm{P} 300$ patients (shaded column). The prolonged P300 patients had significantly lower scores in "orientation" and "memory" than normal P300 patients ("p < 0.05 ).

Our previous study showed that patients with a right hemispheric or midline lesion were more likely to have prolonged $P 300$ latency than those with a left hemispheric lesion. ${ }^{3)}$ Wada et al. ${ }^{15)}$ also reported that right hemispheric lesion correlates with prolonged P300 latency. However, little is known about the influence of changes in rCBF on human P300. ${ }^{16}$ 

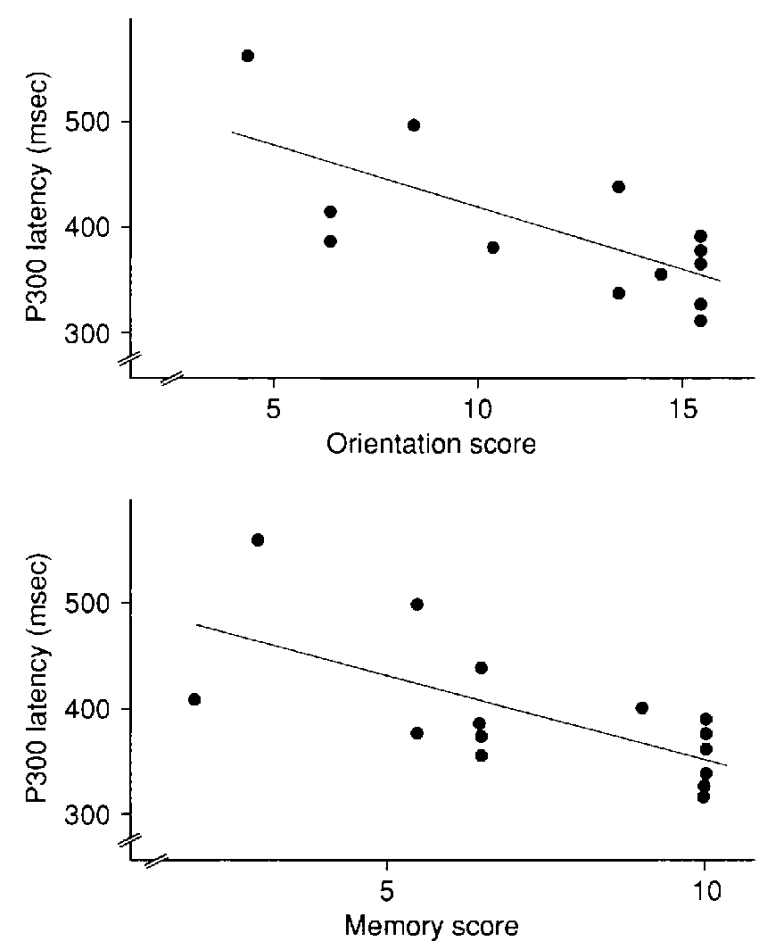

Fig. 9 P300 latency and subtest scores of the patients. There were significant inverse relationships between the P300 latency and scores for "orientation" $(\mathrm{Y}=543.56-12.02 \mathrm{X}, \quad \mathrm{r}=-0.7132$, $\mathrm{p}<0.01)$ and "memory" (Y=518.14 $16.32 X, r=-0.6671, p<0.01$ ).

Our present study focused on the rCBF change in patients with a prolonged $\mathrm{P} 300$ latency to investigate the mechanism by which the P300 becomes abnormal. We found most prolonged P300 patients to have reduced $\mathrm{rCBF}$ in their right cerebral hemisphere, localized primarily in the frontal lobe and/ or thalamus. Moreover, there was a significant correlation between the P 300 latency and the $\mathrm{LI}$ in the frontal lobe. Therefore, decreased right hemispheric rCBF, especially in the frontal lobe and/or thalamus, is associated with prolonged P300 latency. Gainotti ${ }^{4)}$ reported that an indifference reaction is characteristic of patients with right hemispheric lesions. Heilman and Van Den Abell ${ }^{9)}$ suggested that the right hemisphere is dominant for attention. We suggest, therefore, that the right cerebral hemisphere is important in the processes underlying human cognition, and that reduced $\mathrm{rCBF}$ in the right hemisphere may affect the higher brain functions.

rCBF reduction alone does not prolong P300 latency in any given area. In neurosurgical patients, prolonged P300 latency or cognitive defects may also result from metabolic disturbances directly or indi- rectly reducing $\mathrm{rCBF}$. Since there are many neural projections from the thalamus to the frontal lobe, rCBF reduction in the right frontal lobe may be a secondary event resulting from dysfunction of the thalamus. Further research is required to investigate the relationship between rCBF, cerebral metabolism, and the P300 latency throughout the brain.

\section{References}

1) Ebner $\mathrm{A}$, Haas JC, Lucking $\mathrm{CH}$, Schily $\mathrm{M}$, Wallesch $\mathrm{CW}$, Zimmermann P: Event-related brain potentials (P300) and neuropsychological deficit in patients with focal brain lesions. Neurosci Lett 64: 330-334, 1986

2) Folstein MF, Folstein SE, McHugh PR: "Mini-mental State." A practical method for grading the cognitive state of patients for the clinician. $J$ Psychiatr Res 12: 189-198, 1975

3) Funahashi $K$, Maeshima $S$, Kuwata $T$, Nakai $M$, Tsuji N, Komai N: P300 and cognitive functions in cerebrovascular disease, in: The 14th Annual Meeting of the Japanese Cerebrovascular Disease Society (Abstracts). 1989, p 81 (in Japanese)

4) Gainotti G: Emotional behavior and hemispheric side of the lesion. Cortex 8: 41-55, 1972

5) Goodin DS, Starr A, Chippendale T, Squires KC: Sequential changes in the $\mathrm{P} 3$ component of the auditory evoked potential in confusional states and dementing illness. Neurology 33: 1215-1258, 1983

6) Gordon E, Kraiuhin C, Harris A, Meares R, Howson $A$ : The differential diagnosis of dementia using P300 latency. Biol Psychiatry 21: 1123-1132, 1986

7) Gummow LJ, Dustman RE, Keaney RP: Cerebrovascular accident alters P300 event-related potential characteristics. Electroencephalogr Clin Neurophysiol 63: 128-137, 1986

8) Hasegawa $K$, Inoue $K$, Moriya $K$ : An investigation of dementia rating scale for the elderly. Clinical Psychiaty 16: 965-969, 1974 (in Japanese)

9) Heilman KM, Van Den Abell T: Right hemisphere dominance for attention: The mechanism underlying hemispheric asymmetries of inattention (neglect). Neurology 30: 327-330, 1980

10) Knight RT: Decreased response to novel stimuli after prefrontal lesions in man. Electroencephalogr Clin Neurophysiol 59: 9-20, 1984

11) Okada YC, Kaufman L, Williamson SJ: The hippocampal formation as a source of the slow endogenous potentials. Electroencephalogr Clin Neurophysiol 55: 417-426, 1983

12) Prichard WS: Cognitive event-related potential correlates of schizophrenia. Psychol Bull 100: 43-66, 1986

13) Sano $Y$, Tanemura J, Kato M: Rating Scale for Psychological Function for rehabilitation of the patients with brain injury. Rehabilitation Igaku 22: 253, 1985 (in Japanese)

14) Simson R, Vaughan HG Jr, Ritter $W$ : The scalp 
topography of potentials in auditory and visual discrimination tasks. Electroencephalogr Clin Neurophysiol 42: 528-535, 1977

15) Wada S, Yadomi C, Matsukado Y: P300: Its clinical significance in relation to the cerebral lesions and ventricular dilatation. Rinsho Noha 28: 392-398, 1986 (in Japanese)

16) Yamashita K, Kobayashi S, Koide H: P300 eventrelated potentials correlated with cerebral blood flow in patients with cerebral infarction, in: The First International Stroke Congress (Abstracts). 1989, n. pag.

17) Yingling CD, Hosobuchi $Y$ : A subcortical correlate of P300 in man. Electroencephalogr Clin Neurophysiol 59: 72-76, 1984

Address reprint requests to: T. Kuwata, M.D., Department of Neurological Surgery, Wakayama Medical College, 27 7-bancho, Wakayama 640, Japan. 